\title{
Risk Factors for Fracture in Diabetes: The Canadian Multicentre Osteoporosis Study
}

\author{
Lisa-Ann Fraser, ${ }^{1,2}$ Alexandra Papaioannou, ${ }^{3,4}$ Jonathan D. Adachi, ${ }^{4}$ \\ Jinhui $\mathrm{Ma}^{3}$ and Lehana Thabane ${ }^{3,5}$ \\ ${ }^{1}$ Department of Medicine, University of Western Ontario, 339 Windermere Road, London, ON, Canada N6A 5A5 \\ ${ }^{2}$ Division of Endocrinology and Metabolism, St. Joseph's Hospital, 268 Grosvenor Street, London, ON, Canada N6A 4V2 \\ ${ }^{3}$ Department of Epidemiology and Biostatistics, McMaster University, 1280 Main Street West, Hamilton, ON, Canada L8S 4 K1 \\ ${ }^{4}$ Department of Medicine, McMaster University, 1280 Main Street West, Hamilton, ON, Canada L8S 4 K1 \\ ${ }^{5}$ Centre for Evaluation of Medicines, St. Joseph's Healthcare, 50 Charlton Avenue East, Hamilton, ON, Canada L8N 4A6
}

Correspondence should be addressed to Lisa-Ann Fraser; lisaann.fraser@sjhc.london.on.ca

Received 29 July 2013; Accepted 25 August 2013

Academic Editors: N. B. Kandala and C. M. Maylahn

Copyright (C) 2013 Lisa-Ann Fraser et al. This is an open access article distributed under the Creative Commons Attribution License, which permits unrestricted use, distribution, and reproduction in any medium, provided the original work is properly cited.

Objective. Individuals with diabetes have been found to be at increased risk of nontraumatic fracture. However, within the diabetic population, how to distinguish who is at the highest risk and warranting therapy has remained elusive. Design. Cross-sectional analysis of a national population-based cohort study. Patients. Men and women over the age of 50 with diabetes from across Canada. Measurements. Logistic regression analysis to identify diabetes specific factors associated with a history of one or more nontraumatic fractures. Results. Six hundred and six individuals with diabetes with a mean age of 69 years were examined. Thirty percent had a history of non-traumatic fracture. Macrovascular diseases in the form of stroke or TIA, as well as hypertension, were found to be independently associated with fragility fracture. Other, more traditional, clinical risk factors were also associated with fracture, including increased age, female gender, rheumatoid arthritis, family history of osteoporosis, and decreased bone mineral density. Conclusions. In this cohort of Canadians with diabetes, those with rheumatoid arthritis, a family history of osteoporosis, female gender, increased age, decreased BMD, cerebrovascular disease, or hypertension were more likely to have had a non-traumatic fracture. These risk factors may be important to clinicians when identifying which of their diabetic patients are at highest risk of fracture and in need of preventative therapies.

\section{Introduction}

Multiple studies have identified individuals with diabetes as being at increased risk of nontraumatic (fragility) fracture [1, 2]. This is a growing concern as the rates of both diabetes and osteoporosis continue to increase in society [3,4]. In Canada, over 9 million people are living with diabetes or prediabetes, and the number is expected to rise to pandemic levels by 2030 when the global incidence of the disease is predicted to hit 438 million $[4,5]$. Osteoporosis, defined as a $t$-score of $<-2.5$, is also common within the Canadian population with $21 \%$ of Canadian women and 5\% of Canadian men over the age of 50 being affected [6].

Many physicians use bone mineral density (BMD) in addition to fracture to diagnose osteoporosis and to identify individuals in need of therapy for prevention of osteoporotic fractures. In the diabetic population this is a problem. Although type 1 diabetes is often associated with decreased BMD values, those with type 2 diabetes usually have normal or higher than expected BMD values [2]. Similarly, although BMD value is an important risk factor for fragility fracture in the general population, BMD alone does not accurately predict all nontraumatic fractures [7]. In fact, most osteoporotic fractures occur in individuals with BMD values above the osteoporotic range [8]. This understanding has led to a change in focus in recent years, from BMD scores alone to the use of fracture prediction tools to more accurately identify individuals in need of interventions to prevent fragility fractures. The most recent Osteoporosis Canada guidelines, 
published in November 2010, suggest tailoring osteoporosis therapy to individuals with elevated fracture risk identified with fracture prediction tools [9]. Although this approach accurately identifies individuals in need of osteoporosis therapies in the general population, it may not be ideal for those with diabetes. The WHO fracture risk assessment tool (FRAX), a commonly used fracture prediction tool, has been found to underestimate fracture risk in the diabetic population [10].

Diabetes has not been found to alter the predictive power of traditional clinical risk factors for fracture [11]; however, the additional risk incurred by diabetes is not being captured by current prediction tools [10]. As such, physicians treating large populations of diabetic patients are left wondering how to differentiate which of these patients is truly at the greatest risk of fracture and warranting preventative therapy.

We investigated a large population-based cohort, the Canadian Multicenter Osteoporosis study, to explore the diabetic population in order to identify diabetes-specific clinical risk factors associated with a history of fragility fracture.

\section{Methods}

The Canadian Multicentre Osteoporosis Study (CaMos) is an on-going population-based cohort study looking at osteoporosis and fracture risk in community dwelling Canadians. Baseline questionnaires were completed in 1995-1997. The institutional review boards of all sites participating in CaMos approved the study and informed consent was obtained from all participants. The study has been described in detail elsewhere [12], but areas relevant to this study are summarized below. For this analysis, all women and men with diabetes, aged 50 years or older, were included.

2.1. Study Participants. Participants were recruited from within 50-kilometers of one of 9 study centers across Canada (St. John's, Halifax, Quebec City, Toronto, Hamilton, Kingston, Saskatoon, Calgary, and Vancouver). 9,423 individuals (6,539 females and 2,884 males) aged 25 years and older, representing an age-stratified-, sex-, and region-specific sample, were identified from lists of random telephone numbers over an 18-month period.

2.2. Data Collection. A detailed interviewer-administered questionnaire was performed at baseline, which also captured diabetes status. BMD testing was performed, and all participant medications were documented in detail.

2.3. Bone Mineral Density and Fractures. BMD of the hip and lumbar spine (L1-L4) was measured by dual-energy X-ray absorptiometry (DXA) using Hologic QDR 1000, 2000, 4500 or Lunar DPX machines. Densitometers were calibrated daily, and quality assurance was performed following a standard daily and weekly schedule. Cross-calibration of the machines was performed at the nine centers using a European Spine Phantom. BMD results for lumbar spine and femoral neck from all densitometers were converted to a Hologic base using

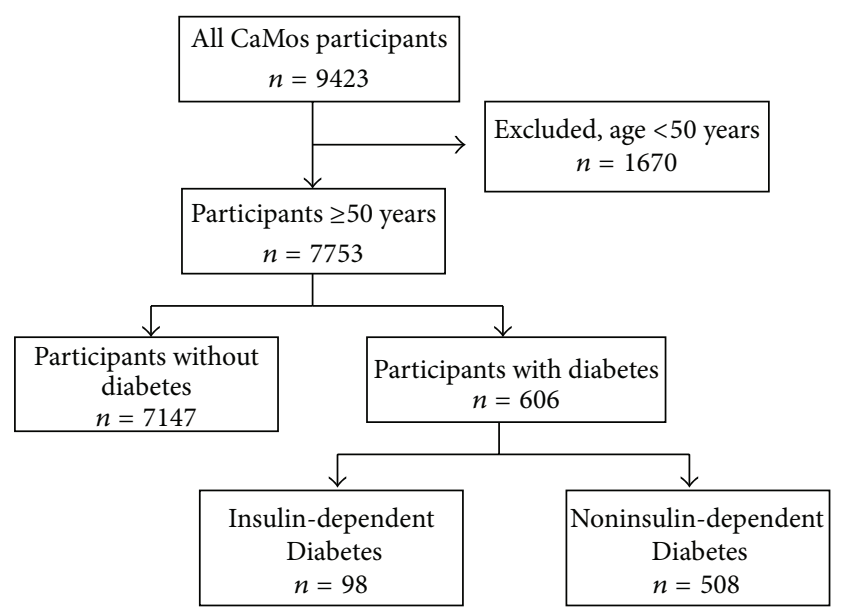

FIGURE 1: Flow diagram of selection of CaMos participants used in analysis.

the method of Genant [13]. All clinically recognized nontraumatic fractures, obtained by self-report, were included in the analysis (hip fractures, clinical vertebral fractures, and nonvertebral fractures). Any fracture associated with trauma or described as a fall from more than standing height was excluded.

2.4. Statistics. The analysis was restricted to CaMos participants $\geq 50$ years who have diabetes. Age was divided into 10-year categories (50-59, 60-69, 70-79, and 80-89). Age 50-59 was used as the reference category in the analyses. Baseline characteristics were described using mean \pm standard deviation. The diabetic subgroup of the CaMos population was examined using a logistic regression model to identify diabetes-specific risk factors for previous nontraumatic fracture. Micro- and macrovascular complications of diabetes have been proposed in the past as possible causes of the increased fracture risk in diabetics [14-16]; therefore these were examined. Variables in the analysis included kidney disease (microvascular disease), macrovascular disease including history of myocardial infarction, stroke, or transient ischemic attack (TIA) hypertension, gender, age, femoral neck $t$-score, rheumatoid arthritis, and family history of osteoporosis. The results are reported as odds ratio (OR), corresponding 95\% confidence interval (CI) and associated $P$ value. The criterion for statistical significance was set at alpha $=0.05$. Multiple imputation was performed for missing data in order to assess the robustness of the model [17]. The results of the model before and after multiple imputation are reported. All calculations were performed using IBM SPSS version 19 (Ireland).

\section{Results}

Of the 7753 CaMos participants over age 50, 606 were identified as having diabetes. Ninety-eight of the individuals with diabetes were on insulin and 508 were not (Figure 1). Characteristics of the baseline CaMos population used in this 
TABLE 1: Baseline characteristics of all CaMos participants over 50 years of age and of participants with diabetes.

\begin{tabular}{lccc}
\hline & $\begin{array}{c}\text { All CaMos participants } \\
>50 \text { yrs }(n=7753)\end{array}$ & $\begin{array}{c}\text { Insulin-dependent } \\
\text { diabetes }(n=98)\end{array}$ & $\begin{array}{c}\text { Noninsulin-dependent } \\
\text { diabetes }(n=508)\end{array}$ \\
\hline Age (years); mean (SD) & $66.7(9.4)$ & $68.0(9.0)$ & $69.4(8.8)$ \\
Gender (female) & $71.8 \%$ & $64.3 \%$ & $65.7 \%$ \\
Femoral neck BMD T-Score; mean (SD) & $-1.24(0.99)$ & $-0.97(1.17)$ & $-0.97(1.04)$ \\
Caucasian & $95.5 \%$ & $93.9 \%$ & $94.3 \%$ \\
Fracture at baseline $(n ; \%)$ & $2133(27.5)$ & $38(38.8)$ & $146(28.7)$ \\
BMI; mean (SD) & $27.1(4.78)$ & $29.73(5.45)$ & $29.06(5.24)$ \\
Years since diagnosis of diabetes; mean; (SD) & N/A & $15.4(11.28)$ & $9.64(9.60)$ \\
Cigarette use ${ }^{*} ; n$ (\%) & $4163(53.7)$ & $54(55.1)$ & $280(55.1)$ \\
Corticosteroid use ${ }^{\dagger} ; n$ (\%) & $415(4.4)$ & $12(12.24)$ & $33(6.50)$ \\
Alcohol use (per wk); mean (SD) & $2.88(5.85)$ & $1.97(5.45)$ & $2.27(6.79)$ \\
\hline * Ever use daily for $>6$ month. & &
\end{tabular}

${ }^{*}$ Ever use daily for $>6$ months.

${ }^{\dagger}$ Oral or IV, ever use daily for $>1$ month.

SD: standard deviation, BMD: bone mineral density, and BMI: body mass index.

TABLE 2: Variables associated with a history of non-traumatic fracture in diabetic participants in CaMos $>50$ years of age.

\begin{tabular}{|c|c|c|c|c|c|c|}
\hline \multirow{2}{*}{ Variable } & \multicolumn{3}{|c|}{ Without multiple imputation; $n=335$} & \multicolumn{3}{|c|}{ With multiple imputation; $n=597$} \\
\hline & Odds ratio & 95\% Confidence interval & $P$ value & Odds ratio & 95\% Confidence interval & $P$ value \\
\hline Gender (female) & 1.03 & $0.89-1.20$ & 0.686 & 1.13 & $1.00-1.27$ & 0.045 \\
\hline \multicolumn{7}{|l|}{ Age } \\
\hline $60-69$ yrs & 1.12 & $0.95-1.33$ & 0.166 & 1.07 & $0.93-1.22$ & 0.370 \\
\hline $70-79$ yrs & 1.35 & $1.13-1.61$ & 0.001 & 1.20 & $1.03-1.39$ & 0.017 \\
\hline $80-89$ yrs & 1.35 & $1.01-1.91$ & 0.044 & 1.03 & $0.83-1.27$ & 0.793 \\
\hline FN T-Score (increasing score) & 0.76 & $0.71-0.82$ & $<0.001$ & 0.79 & $0.75-0.84$ & $<0.001$ \\
\hline Family history of osteoporosis & 1.36 & $1.16-1.61$ & $<0.001$ & 1.27 & $1.10-1.46$ & 0.001 \\
\hline Rheumatoid arthritis & 1.40 & $1.09-1.79$ & 0.008 & 1.30 & $1.07-1.59$ & 0.009 \\
\hline Stroke or TIA & 1.65 & $1.22-2.23$ & 0.001 & 1.51 & $1.20-1.91$ & $<0.001$ \\
\hline Myocardial infarction & 1.21 & $0.94-1.57$ & 0.139 & 1.10 & $0.90-1.33$ & 0.347 \\
\hline Hypertension & 1.11 & $0.96-1.28$ & 0.159 & 1.16 & $1.04-1.29$ & 0.010 \\
\hline Kidney disease & 1.30 & $0.79-2.13$ & 0.296 & 1.09 & $0.73-1.61$ & 0.681 \\
\hline
\end{tabular}

FN: femoral neck; TIA: transient ischemic attack.

analysis are displayed in Table 1 . The diabetic population was $65 \%$ female and $94 \%$ Caucasian, with a mean age (standard deviation (SD)) of 69.1 (8.8) years, mean (SD) body mass index (BMI) of 29.2 (5.3), and femoral neck BMD $t$-score of -0.97 (1.06). Those requiring insulin had been diagnosed with diabetes $15.4(\mathrm{SD}=11.28)$ years ago. Those not requiring insulin had diabetes for 9.64 (9.6) years. No patients were on thiazolidinediones at study baseline. Of the 606 individuals with diabetes, $184(30 \%)$ had a history of previous fragility fracture. History of a stroke or TIA (macrovascular disease) was found to be associated with a history of nontraumatic fracture (OR: 1.65; 95\% CI 1.22, 2.23; $P=0.001$ ) (Table 2). Family history of osteoporosis, age greater than 70 years, rheumatoid arthritis, and low femoral neck $t$-score were also more likely to be found in the group of diabetics that had a history of fracture. After multiple imputation for missing data, the number of diabetics included in the analysis increased significantly. History of cerebrovascular disease (in the form of a stroke or TIA) was still found to be associated with fracture amongst diabetics (OR: 1.51; 95\% CI 1.20, 1.91;
$P=0.001)$ but hypertension was also identified as being associated with fracture amongst diabetics (OR: 1.16; 95\% CI 1.04, 1.29; $P=0.010)$. An interaction term for stroke and hypertension was not significant. Other risk factors found to be important within the diabetic population were rheumatoid arthritis, family history of osteoporosis, older age, female gender, and BMD. Duration of diabetes was not significant when added as a variable.

\section{Discussion}

We found an association between cerebrovascular disease and hypertension and a history of nontraumatic fracture in Canadian diabetics. In addition to these, we also reaffirmed some traditional osteoporosis clinical risk factors (age, BMD, family history of osteoporosis, and rheumatoid arthritis) in a Canadian diabetic population.

Traditional fracture risk factors, such as those used in FRAX, have been studied only to a limited degree within the diabetic population. Significant differences in fracture 
pathophysiology exist between diabetic and nondiabetic populations $[18,19]$, and therefore it cannot be assumed that fracture risk associated with traditional risk factors can be extrapolated to the diabetic population. To our knowledge, there are currently just two studies examining traditional clinical fracture risk factors in individuals with diabetes [11, 20]. The first study identified age, BMI, prior fracture, secondary osteoporosis, and corticosteroid use to be associated with increased fracture risk in type 2 diabetics in Rochester Minnesota. The second study looked at women with diabetes in Manitoba Canada and identified prior fracture, age, BMI, and BMD as important. Our study expands the list of traditional risk factors to include rheumatoid arthritis and family history of osteoporosis as risk factors within the diabetic population that are associated with increased risk of nontraumatic fracture.

We further examined diabetes-specific risk factors and found diabetics with cerebrovascular disease or hypertension to be at increased risk of nontraumatic fracture. Cerebrovascular disease (stroke, TIA) is known to be associated with fragility fractures in the general population [21]. Immobilization and vitamin D deficiency often lead to decreased BMD in these individuals [22], and residual problems with balance and dizziness predispose them to falls [23]. To our knowledge, the only study to-date finding stroke as a risk factor for fracture specifically in the diabetic population is an Ontario-based study which found increased hip fracture risk in type 2 diabetics with a history of previous stroke [14]. Our study expands stroke as a risk factor in diabetics for any nontraumatic fracture, not just hip. Hypertension is not widely recognized as a fracture risk factor. However, recent studies have identified an association between high blood pressure and bone fragility. A population-based study from Denmark found increased fracture risk (in a nondiabetic population) following a diagnosis of hypertension [24]. This increased risk was found to be independent of stroke or myocardial infarction and independent of drug effects. In other studies, multiple different hypertension medications have been found to decrease fracture risk, supporting a link between blood pressure elevation and risk of fracture [25]. However, increased risk of hip fracture in elderly patients immediately after initiating antihypertensive medications has also been reported, likely secondary to increased falls [26]. There have also been studies showing an association between hypertension and the surrogate outcome of increased BMD loss [27]. This association may be mediated by increased urinary calcium losses in hypertensive individuals $[28,29]$. Our study adds important information to the literature, arming clinicians with the knowledge that previous stroke/TIA and hypertension further increase the risk of fracture in diabetic patients. This knowledge can be an important tool to help practitioners differentiate risk in their diabetic patients and hence appropriately focus preventative strategies.

There are several previous studies focusing on factors specific to diabetes, rather than traditional clinical risk factors for fracture, but results have been variable. Increased BMI and centripetal obesity, which are commonly seen in type 2 diabetes, have been found in some studies to have a protective effect, increasing BMD and decreasing the risk of fracture
[30, 31]. Risk of fracture has been found to increase with the duration of diabetes [32], possibly due to the increased incidence of micro- and macrovascular complications that occur with time. These complications include retinopathy, neuropathy, peripheral vascular disease, stroke, and myocardial infarction, all of which have been linked to increased fracture risk [14-16]. It is possible that the associations between these diabetic complications and fracture risk are mediated by an increased fall risk seen in severe disease [15]. Hyperglycemia has been found in multiple studies to be associated with increased fracture risk $[16,18]$. Insulin use has also been associated with increased fracture risk in some studies [16], possibly representing a marker of more severe, long-standing disease in type 2 diabetes. Insulin also predisposes patients to hypoglycemic episodes and therefore increased fall risk. However, other studies have found a decreased fracture risk in diabetics using insulin [33], perhaps relating to the fact that insulin has an important anabolic effect on bone. Likewise, insulin therapy has been found to be associated with enhanced bone formation [19] and the hyperinsulinemia associated with early type 2 diabetes may even be protective against fracture [33]. Sulfonylurea medications and metformin have been associated with a decrease fracture risk [33] whereas rosiglitazone has been found to be associated with a 2 -fold increase in fractures [34].

Strengths of our study include the use of a national population-based cohort, bone mineral density measurement, using fracture as an outcome, and investigation of novel diabetes-specific fracture risk factors. However, several limitations apply. As a retrospective study, we can only identify associations between events as we do not know if stroke, hypertension, and so forth occurred before or after participants' nontraumatic fracture. Another limitation was our inability to distinguish between type 1 and type 2 diabetes within our population. However, only $9.6 \%$ of the diabetic population that was on insulin reported being diagnosed with diabetes at an age of less than 30 years. As such, our diabetic population was presumably made up of mostly type 2 diabetics, an assumption that has been made in other large database studies of diabetic patients [14, 32]. The high BMD and BMI values measured in our diabetic population (Table 1) support the assumption of a predominance of type 2 diabetes. All the CaMos questionnaires depended on patient reporting (and therefore are subject to patient recall bias and misunderstanding); therefore the incidence of certain classically underdiagnosed conditions (such as hyperglycemia and hypertension) is likely grossly underestimated. However, bias from undiagnosed diabetes and hypertension would be expected to, if anything, decrease the effect sizes found. Lastly, we were unable to explore diabetic retinopathy, peripheral neuropathy, or peripheral vascular disease as fracture risk factors as data relating to these conditions were not collected as part of the CaMos study.

\section{Conclusions}

Amongst this group of Canadians with diabetes, those with rheumatoid arthritis, a family history of osteoporosis, female 
gender, increased age, decreased BMD, cerebrovascular disease, or hypertension were more likely to have had a nontraumatic fracture. These risk factors may therefore be important to clinicians to identify which of their patients with diabetes are at highest fracture risk and deserve particular attention for preventative action.

\section{Disclosure}

Lisa-Ann Fraser is on the speakers bureau for Amgen. Alexandra Papaioannou is a consultant/speaker for Amgen, Aventis, Eli Lilly, Merck Frosst, Novartis, Procter \& Gamble, Servier, and Wyeth-Ayerst, has conducted clinical trials for Eli Lilly, Merck Frosst, Novartis, Procter \& Gamble, and SanofiAventis, and received unrestricted grants from Amgen, Eli Lilly, Merck Frosst, Procter and Gamble, and Sanofi-Aventis. Jonathan D. Adachi has received research support from, and is a consultant for, Amgen, Astra Zeneca, Eli Lilly, GlaxoSmithKline, Merck, Novartis, Nycomed, Pfizer, Procter and Gamble, Roche, Sanofi Aventis, Servier, Warner Chilcott, Wyeth. Jinhui Ma and Lehana Thabane have no disclosure.

\section{Conflict of Interests}

The authors have no conflicting interests with the current study.

\section{Acknowledgments}

The authors thank all the participants in the CaMos study whose participation made this research possible. The CaMos Research Group are David Goltzman (coprincipal investigator, McGill University), Nancy Kreiger (coprincipal investigator, Toronto), and Alan Tenenhouse (principal investigator emeritus, Toronto). Investigators from CaMos Coordinating Centre, McGill University, Montreal, Quebec, are Suzette Poliquin (national coordinator), Suzanne Godmaire (research assistant), Silvia Dumont (administrative assistant), Claudie Berger (study statistician), and Wei Zhou (statistician). Participants from Memorial University, St. John's Newfoundland, are Carol Joyce (director), Christopher Kovacs (codirector), and Emma Sheppard (coordinator). Participants from Dalhousie University, Halifax, Nova Scotia, are Susan Kirkland, Stephanie Kaiser (codirectors), and Barbara Stanfield (coordinator). Participants from Laval University, Quebec City, Quebec, are Jacques P. Brown (director), Louis Bessette (codirector), and Marc Gendreau (coordinator). Participants from Queen's University, Kingston, Ontario are Tassos Anastassiades (director), Tanveer Towheed (codirector), and Barbara Matthews (coordinator). Participants from University of Toronto, Toronto, Ontario, are Bob Josse (director), Sophie Jamal (codirector), Tim Murray (past director), and Barbara Gardner-Bray (coordinator). Participants from McMaster University, Hamilton, Ontario, are Jonathan D. Adachi (director), Alexandra Papaioannou (codirector), and Laura Pickard (coordinator). Participants from University of Saskatchewan, Saskatoon, Saskatchewan, are Wojciech P. Olszynski (director), K. Shawn Davison (codirector), and
Jola Thingvold (coordinator). Participants from University of Calgary, Calgary, Alberta, are David A. Hanley (director) and Jane Allan (coordinator). Participants from University British Columbia, Vancouver, British Columbia, are Jerilynn C. Prior (director), Millan Patel (codirector), Yvette Vigna (coordinator), and Brian Lentle (radiologist). The Canadian Multicentre Osteoporosis Study was funded by the Canadian Institutes of Health Research (CIHR); Merck Frosst Canada Ltd.; Eli Lilly Canada Inc.; Novartis Pharmaceuticals Inc.; The Alliance: sanofi-aventis and Procter and Gamble Pharmaceuticals Canada Inc.; Servier Canada Inc.; Amgen Canada Inc.; The Dairy Farmers of Canada; and The Arthritis Society.

\section{References}

[1] M. Janghorbani, R. M. van Dam, W. C. Willett, and F. B. Hu, "Systematic review of type 1 and type 2 diabetes mellitus and risk of fracture," American Journal of Epidemiology, vol. 166, no. 5, pp. 495-505, 2007.

[2] P. Vestergaard, "Discrepancies in bone mineral density and fracture risk in patients with type 1 and type 2 diabetes-a metaanalysis," Osteoporosis International, vol. 18, no. 4, pp. 427-444, 2007.

[3] D. A. Hanley and R. G. Josse, "Prevention and management of osteoporosis: consensus statements from the Scientific Advisory Board of the Osteoporosis Society of Canada. 1. Introduction," Canadian Medical Association Journal, vol. 155, no. 7, pp. 921-923, 1996.

[4] P. Hossain, B. Kawar, and M. El Nahas, "Obesity and diabetes in the developing world-a growing challenge," The New England Journal of Medicine, vol. 356, no. 3, pp. 213-215, 2007.

[5] Canadian Diabetes Association, "The prevalence and costs of diabetes," 2013, http://www.diabetes.ca/diabetes-and-you/ what/prevalence/.

[6] C. Berger, D. Goltzman, L. Langsetmo et al., "Peak bone mass from longitudinal data: implications for the prevalence, pathophysiology, and diagnosis of osteoporosis," Journal of Bone and Mineral Research, vol. 25, no. 9, pp. 1948-1957, 2010.

[7] W. D. Leslie, C. Metge, and L. Ward, "Contribution of clinical risk factors to bone density-based absolute fracture risk assessment in postmenopausal women," Osteoporosis International, vol. 14, no. 4, pp. 334-338, 2003.

[8] A. Cranney, S. A. Jamal, J. F. Tsang, R. G. Josse, and W. D. Leslie, "Low bone mineral density and fracture burden in postmenopausal women," Canadian Medical Association Journal, vol. 177, no. 6, pp. 575-580, 2007.

[9] A. Papaioannou, S. Morin, A. M. Cheung et al., "2010 clinical practice guidelines for the diagnosis and management of osteoporosis in Canada: summary," Canadian Medical Association Journal, vol. 182, no. 17, pp. 1864-1873, 2010.

[10] L. M. Giangregorio, W. D. Leslie, L. M. Lix et al., "FRAX underestimates fracture risk in patients with diabetes," Journal of Bone and Mineral Research, vol. 27, no. 2, pp. 301-308, 2012.

[11] L.-A. Fraser, J. Pritchard, G. Ioannidis et al., "Clinical risk factors for fracture in diabetes: a matched cohort analysis," Journal of Clinical Densitometry, vol. 14, no. 4, pp. 416-421, 2011.

[12] N. Kreiger, A. Tenenhouse, L. Joseph et al., "The Canadian Multicentre Osteoporosis Study (CaMos): background, rationale, methods," Canadian Journal on Aging, vol. 18, no. 3, pp. 376387, 1999. 
[13] H. K. Genant, S. Grampp, C. C. Glüer et al., "Universal standardization for dual x-ray absorptiometry: patient and phantom cross-calibration results," Journal of Bone and Mineral Research, vol. 9, no. 10, pp. 1503-1514, 1994.

[14] L. L. Lipscombe, S. A. Jamal, G. L. Booth, and G. A. Hawker, "The risk of hip fractures in older individuals with diabetes: a population-based study," Diabetes Care, vol. 30, no. 4, pp. 835841, 2007.

[15] S. Patel, S. Hyer, K. Tweed et al., "Risk factors for fractures and falls in older women with type 2 diabetes mellitus," Calcified Tissue International, vol. 82, no. 2, pp. 87-91, 2008.

[16] R. Q. Ivers, R. G. Cumming, P. Mltchell, and A. J. Peduto, "Diabetes and risk of fracture: the blue mountains eye study," Diabetes Care, vol. 24, no. 7, pp. 1198-1203, 2001.

[17] D. B. Rubin, Multiple Imputation, John Wiley \& Sons, New York, NY, USA, 1989.

[18] D. Merlotti, L. Gennari, F. Dotta, D. Lauro, and R. Nuti, "Mechanisms of impaired bone strength in type 1 and 2 diabetes," Nutrition, Metabolism and Cardiovascular Diseases, vol. 20, no. 9, pp. 683-690, 2010.

[19] L. R. McCabe, "Understanding the pathology and mechanisms of type I diabetic bone loss," Journal of Cellular Biochemistry, vol. 102, no. 6, pp. 1343-1357, 2007.

[20] L. J. Melton III, C. L. Leibson, S. J. Achenbach, T. M. Therneau, and S. Khosla, "Fracture risk in type 2 diabetes: update of a population-based study," Journal of Bone and Mineral Research, vol. 23 , no. 8, pp. 1334-1342, 2008.

[21] A. Ramnemark, L. Nyberg, B. Borssén, T. Olsson, and Y. Gustafson, "Fractures after stroke," Osteoporosis International, vol. 8, no. 1, pp. 92-95, 1998.

[22] É. Smith and Á. Carroll, "Bone mineral density in adults disabled through acquired neurological conditions: a review," Journal of Clinical Densitometry, vol. 14, no. 2, pp. 85-94, 2011.

[23] S. E. Lamb, L. Ferrucci, S. Volapto, L. P. Fried, and J. M. Guralnik, "Risk factors for falling in home-dwelling older women with stroke: the women's health and aging study," Stroke, vol. 34, no. 2, pp. 494-501, 2003.

[24] P. Vestergaard, L. Rejnmark, and L. Mosekilde, "Hypertension is a risk factor for fractures," Calcified Tissue International, vol. 84, no. 2, pp. 103-111, 2009.

[25] L. Rejnmark, P. Vestergaard, and L. Mosekilde, “Treatment with beta-blockers, ACE inhibitors, and calcium-channel blockers is associated with a reduced fracture risk: a nationwide casecontrol study," Journal of Hypertension, vol. 24, no. 3, pp. 581589, 2006.

[26] D. A. Butt, M. Mamdani, P. C. Austin et al., "The risk of hip fracture after initiating antihypertensive drugs in the elderly," Archives of Internal Medicine, vol. 172, pp. 1739-1744, 2012.

[27] F. P. Cappuccio, E. Meilahn, J. M. Zmuda, and J. A. Cauley, "High blood pressure and bone-mineral loss in elderly white women: a prospective study," The Lancet, vol. 354, no. 9183, pp. 971-975, 1999.

[28] P. Strazzullo, "The renal calcium leak in primary hypertension: pathophysiological aspects and clinical implications," Nutrition, Metabolism \& Cardiovascular Diseases, vol. 1, pp. 98-103, 1991.

[29] D. A. McCarron, P. A. Pingree, and R. J. Rubin, "Enhanced parathyroid function in essential hypertension: a homeostatic response to a urinary calcium leak," Hypertension, vol. 2, no. 2 , pp. 162-168, 1980.

[30] I. Kanazawa, T. Yamaguchi, M. Yamamoto, M. Yamauchi, S. Yano, and T. Sugimoto, "Combination of obesity with hyperglycemia is a risk factor for the presence of vertebral fractures in type 2 diabetic men," Calcified Tissue International, vol. 83, no. 5, pp. 324-331, 2008.

[31] T. Yamaguchi, I. Kanazawa, M. Yamamoto et al., "Associations between components of the metabolic syndrome versus bone mineral density and vertebral fractures in patients with type 2 diabetes," Bone, vol. 45, no. 2, pp. 174-179, 2009.

[32] W. D. Leslie, L. M. Lix, H. J. Prior, S. Derksen, C. Metge, and J. O'Neil, "Biphasic fracture risk in diabetes: a population-based study," Bone, vol. 40, no. 6, pp. 1595-1601, 2007.

[33] P. Vestergaard, L. Rejnmark, and L. Mosekilde, "Relative fracture risk in patients with diabetes mellitus, and the impact of insulin and oral antidiabetic medication on relative fracture risk," Diabetologia, vol. 48, no. 7, pp. 1292-1299, 2005.

[34] S. E. Kahn, B. Zinman, J. M. Lachin et al., "Rosiglitazone associated fractures in type 2 diabetes: an analysis from A Diabetes Outcome Progression Trial (ADOPT)," Diabetes Care, vol. 31, no. 5, pp. 845-851, 2008. 


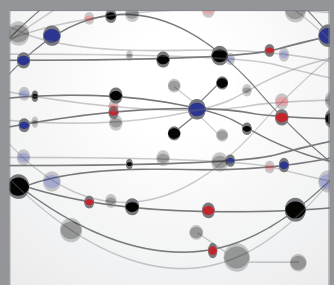

The Scientific World Journal
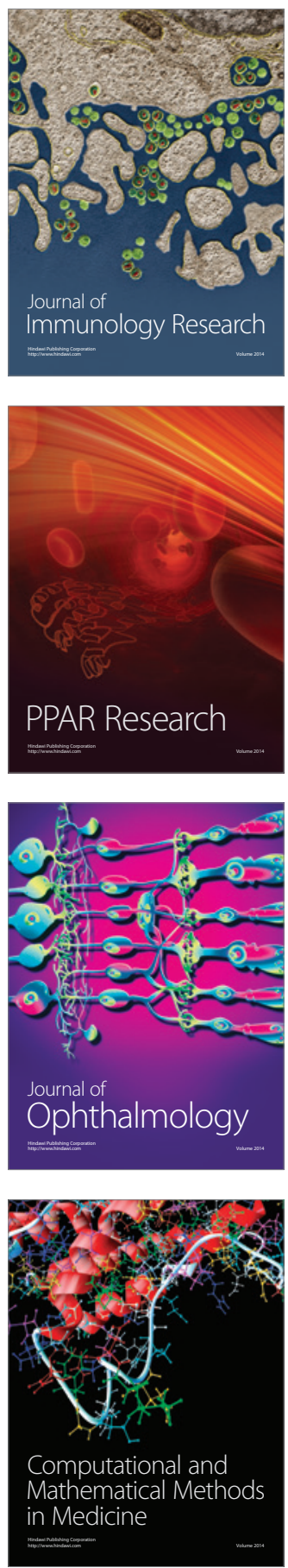

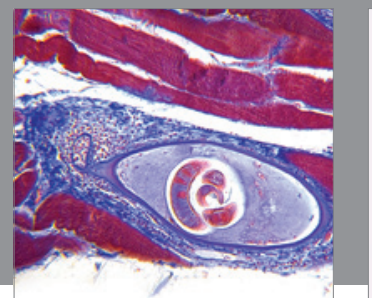

Gastroenterology

Research and Practice
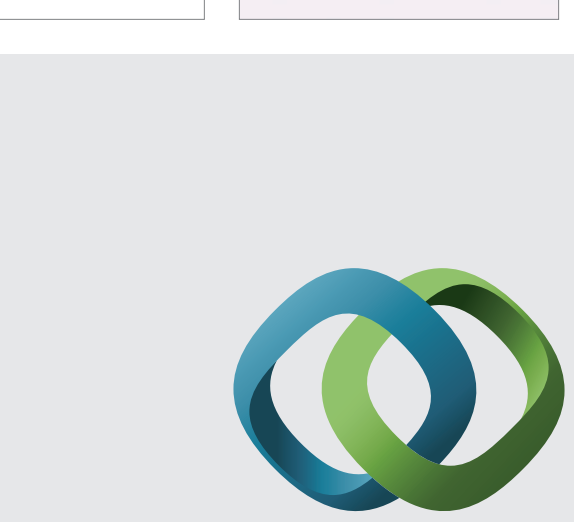

\section{Hindawi}

Submit your manuscripts at

http://www.hindawi.com
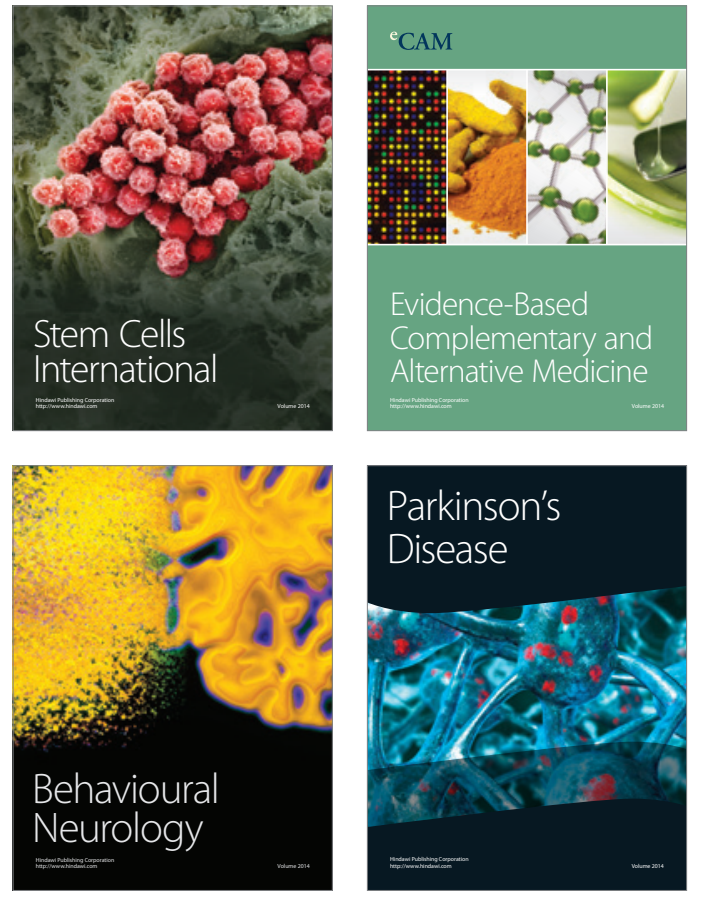
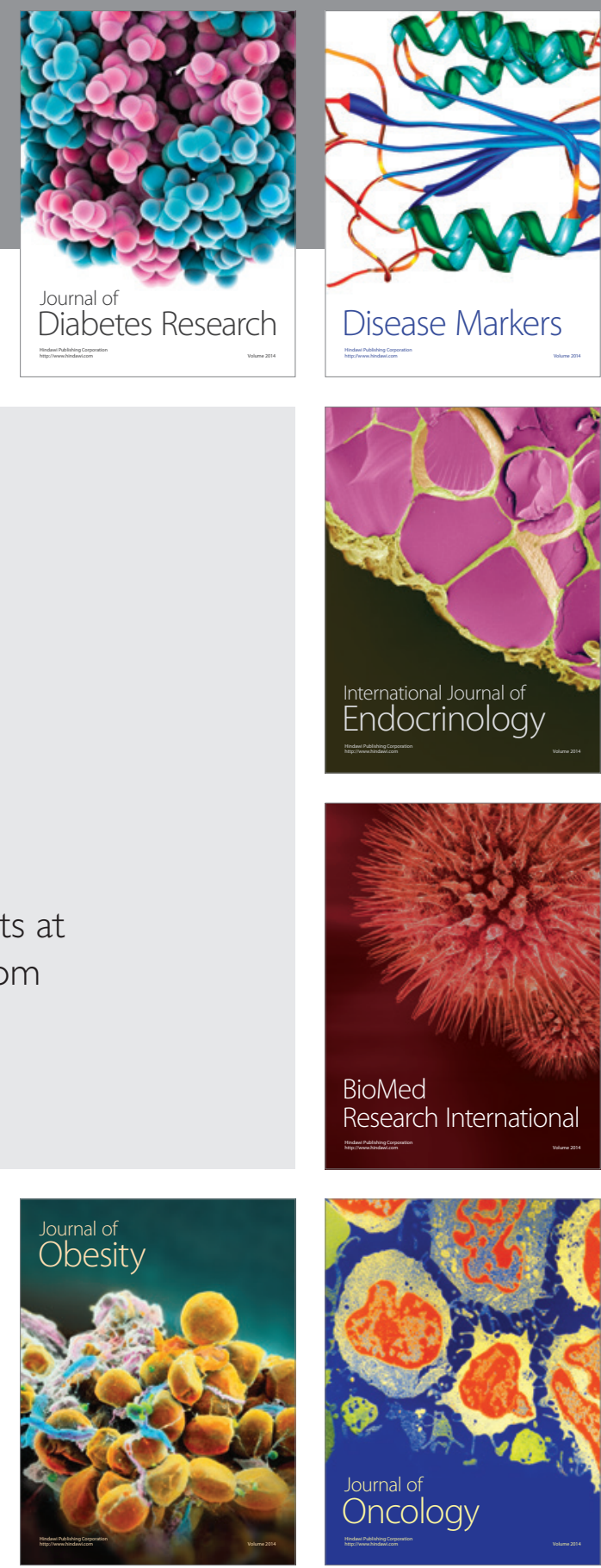

Disease Markers
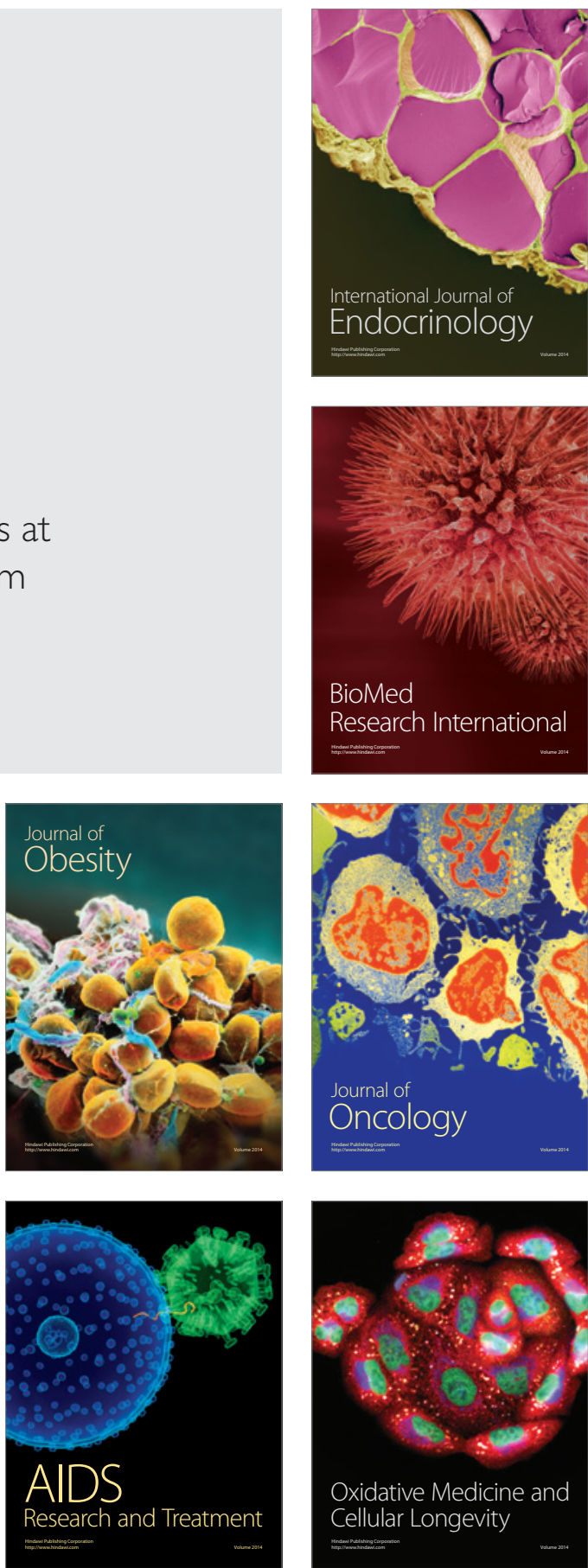